\title{
Preface
}

\author{
Pierre Bonami • Leo Liberti • Andrew J. Miller • \\ Annick Sartenaer
}

This issue of Mathematical Programming B contains invited papers from authors who participated in the European Workshop on Mixed Integer Nonlinear Programming (EWMINLP), held at the Centre International de Rencontres Mathématiques in Luminy near Marseille, France, during April 12-16, 2010. Information about EWMINLP, including the book of proceedings, can be found at https://sites.google. com/site/ewminlp/. EWMINLP is the second of a series of workshops on MINLP, which started with the "Hot Topics Workshop" at the Institute of Mathematics and Applications (IMA) in Minneapolis in 2008, and continued with the Exploratory Workshop on MINLP in Sevilla, at the end of 2010. (Of these, EWMINLP was the only one in which many of the participants had their travel arrangements disrupted by volcano ash; the eruptions of Eyjafjallajökull beginning on April 14 prevented many of our North American participants from returning home for close to a week.) At present there are two candidates for the next editions: Pittsburgh and Rio de Janeiro. But no dates have been set yet.

P. Bonami

LIF, CNRS Université d'Aix Marseille, 163 Av. de Luminy, 13009 Marseille, France

e-mail: pierre.bonami@lif.univ-mrs.fr

L. Liberti

LIX, École Polytechnique, 91128 Palaiseau, France

e-mail: liberti@lix.polytechnique.fr

\section{A. J. Miller}

Institut de Mathématiques de Bordeaux, Université de Bordeaux 1, RealOpt, INRIA Bordeaux Sud-Ouest, Bordeaux, France

e-mail: andrew.miller@math.u-bordeaux1.fr

\section{A. Sartenaer $(\varangle)$}

naXys, University of Namur (FUNDP), 5000 Namur, Belgium

e-mail: annick.sartenaer@fundp.ac.be 
MINLP models are a set of Mathematical Programming (MP) formulations involving both integer and continuous definition domains for the decision variables, and both linear and nonlinear terms in the objective function and constraints. MINLP problems present themselves in a wide variety of application fields, such as chemical process design (nonlinearities express the balance of mass and energy in containers with complicated geometries, and integer variables model setup decisions and allocations), water network routing and planning (nonlinearities occur because of pressure varying nonlinearly at the joints, while pipe diameters and the location of certain equipment are discrete decisions), air traffic management (nonlinearities occur because of the flight trajectories, and binary variables are used to model early/late arrivals and disjunctive constraints), and others. The term "MINLP" was probably first used by I. Grossmann, but he claims his Ph.D. advisor R. Sargent used the phrase "mixed-integer nonlinear programming" verbally; at the time (mid-seventies), there were no practical methods for solving MINLP problems.

Since then many theoretical and experimental advances have been made, and today numerous techniques are available and implemented in general purpose solvers. Nevertheless, the state of the art is limited, and MINLP still stands as one of the most challenging areas of optimization. The last decade has seen a renewed interest in MINLP. In particular, specialists from different fields of optimization (global, nonlinear, integer, combinatorial, etc.) have collaborated and contributed new ideas to MINLP by building on recent advances in their respective fields. It was the spirit, and goal, of the EWMINLP workshop in Marseille to bring together a group of such specialists with very diverse backgrounds who could contribute new ideas to the field. We hope that this issue reflects this diversity and can appeal to all researchers in Mathematical Optimization. May it encourage each of us to take our chances in solving MINLP problems (Fig. 1)!

Here is an overview of the contents of this issue (by alphabetical order of the title):

- On Convex Relaxations for Quadratically Constrained Quadratic Programming, by Kurt Anstreicher, compares relaxations based on convex lower envelopes, differences of convex underestimators, RLT constraints, and SDP relaxations. It shows in particular that a combination of RLT and SDP constraints dominates the other approaches.

- On Semidefinite Programming Relaxations of Maximum k-section, by Etienne de Klerk, Dmitrii Pasechnik, Renata Sotirov, and Cristian Dobre, proposes a new SDP bound for the maximum k-section problem that is particularly appropriate for graphs with suitable symmetry.

- Numerical Study of Semidefinite Bounds for the k-cluster Problem, by Jerome Malick and Frédéric Roupin, presents a novel branch-and-bound algorithm for solving the aforementioned problem. The algorithm is based on a new SDP-based bounding procedure.

- The Quadratic Graver Cone, Quadratic Integer Minimization, and Extensions, by Jon Lee, Shmuel Onn, Lyubov Romanchuk, and Robert Weismantel, deals with problems of the form $\min \left\{f(x): A x=b, l \leq x \leq u, x \in Z^{n}\right\}$. It is shown that Graver bases can be used to minimize $f$ when it is quadratic or a higher degree polynomial function lying in suitable cones. 


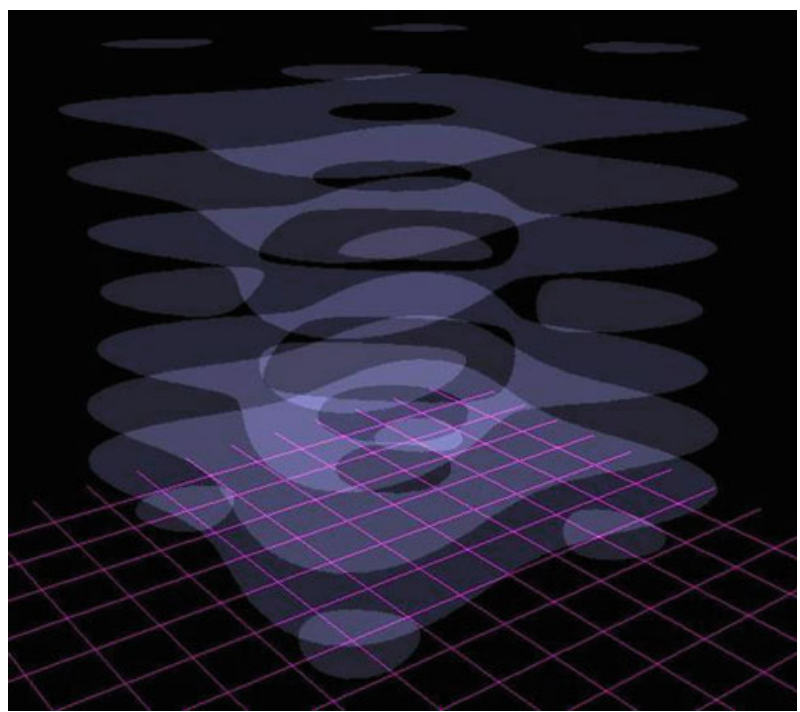

Fig. 1 A representation of a MINLP problem. With the kind permission of Thorsten Gellermann

- Some Results on the Strength of Relaxations of Multilinear Functions, by James Luedtke, Mahdi Namazifar, and Jeff Linderoth, presents approximation results for relaxations obtained by approximating the convex hull of general multilinear functions using only the convex hull of the product of two variables. Surprisingly, in many cases the approximation obtained is provably quite good. It is in particular a constant factor for general bilinear functions.

- SpeeDP:An Algorithm to Compute SDP Bounds for Very Large Max-Cut Instances, by Luigi Grippo, Laura Palagi, Mauro Piacentini, Veronica Piccialli, and Giovani Rinaldi, presents a new nonlinear programming based algorithm for solving the famous SDP relaxation of the Max-Cut problem. The algorithm is tested empirically and used in a variant of the Goemans-Williamson algorithm, and it is able to find near-optimal solutions for Max-Cut instances with millions of nodes.

- A Storm of Feasibility Pumps for Nonconvex MINLP, by Claudia D'Ambrosio, Antonio Frangioni, Leo Liberti, and Andrea Lodi, aims at generalizing the feasibility pump heuristic method to the setting of nonconvex MINLPs. Extensive computational tests show that the new method is able to find feasible solutions for many difficult instances from the literature. 